\title{
A study of the spatial variation of electric field in highly resistive metal films by scanning tunneling potentiometry
}

\author{
G. Ramaswamy, A.K. Raychaudhuri, K. Das Gupta, G. Sambandamurthy \\ Department of Physics, Indian Institute of Science, Bangalore 560 012, India \\ (Fax: +91-080/3341683, E-mail: geetha@physics.iisc.ernet.in (G. R.), arup@physics.iisc.ernet.in (A.K. R.)) \\ Received: 25 July 1997/Accepted: 1 October 1997
}

\begin{abstract}
Electronic transport in highly resistive (but metallic) thin platinum films $(\approx 10 \mathrm{~nm})$ deposited by electronbeam evaporation has been studied by scanning tunneling microscopy and scanning tunneling potentiometry (STP). The films have an average grain size of $\approx 10 \mathrm{~nm}$. On this scale transport through the film is very inhomogeneous. Scattering from grain boundaries (GBs) results in large variations in the local potential resulting in fields as high as $10^{4}-10^{5} \mathrm{~V} / \mathrm{cm}$ located near the GB. The reflection coefficient $R_{\mathrm{g}}$ of electrons at a GB has values between $0.5-0.7$ as determined from the $\mathrm{STP}$ data. This can be compared to an average $\left\langle R_{\mathrm{g}}\right\rangle \approx 0.9 \mathrm{ob}-$ tained from an analysis of the bulk resistivity data taken over the temperature range $4.2 \mathrm{~K}<T<300 \mathrm{~K}$.
\end{abstract}

Charge transport in thin metal films is not uniform on the nanometer scale and is accompanied by strong inhomogeneities in the field and current density near localized scatterers such as grain boundaries (GBs) and defects. Conventional theories of transport ignore these spatial variations and consider the averaged field. In this average picture all details of the charge transport in the conductor are parameterized by a single parameter, namely the mean free path of the charge carrier. This describes the correct picture in a bulk system but as the sample dimension decreases it becomes necessary to measure the relevant quantities on a nanometer scale and the deviations of the local fields and potentials from the average values become significant. The significance of the influence of localized scatterers was first recognized and investigated theoretically by Landauer [1]. He pointed out that the microscopic dipole field that results from the asymmetry of the electron scattering at a point defect is the ultimate source of the residual resistivity. Work of Landauer and subsequent developments [2,3] showed the equivalence of the residual resistivity dipole approach and the more conventional Boltzmann transport approach. Understanding the exact microscopic nature of the scattering process is important in order to gain insight into fundamental questions like charge transport at the nanometer scale and is also of practical interest in phenomena like electromigration. While bulk transport properties which give a measure of the average behavior have been investigated for a long time, the actual electronic processes taking place at a nanometer level (at the scale of electronic mean free path) remained essentially unanswered mainly because of the lack of an experimental tool to study the properties on this scale. Development of scanning probe microscopies (SPMs) $[4,5]$ and in particular scanning tunneling potentiometry (STP) [6-8] has enabled us to experimentally observe the scattering-induced field variations at a nanometer scale. More recent theoretical studies have established the condition under which scanning tunneling microscopy (STM) can measure the change in local electrochemical potential [9]. It is only recently with the advent of STP that this issue is being reinvestigated and a few experimental studies have been reported $[10,11]$.

In this paper we report the direct observation of potential steps and the build up of a field at the nanometer scale at GBs on thin metal films using STM and STP. We also examine the correlation of physical parameters measured at a nanometer level with those obtained from bulk measurements. Although these films show a clear metallic positive temperature coefficient of resistivity, they are highly resistive and are almost on the verge of forming an island-type structure and can be considered to be on the metallic side close to the percolation threshold.

\section{Experimental details}

The scanning tunneling microscope used in the experiment was built in-house and has a design consisting of two concentric piezoelectric tubes. The outer tube is sectored to provide the $X-Y$ scan and carries the sample. The inner tube provides $Z$ positioning of the tip. The outer and inner tubes have gains of $9.0 \mathrm{~nm} / \mathrm{V}$ and $4.5 \mathrm{~nm} / \mathrm{V}$ respectively. The tipsample coarse approach is by a mechanical system consisting of a fine pitch screw and a worm gear giving a linear motion of $\approx 25 \mathrm{~nm} /$ degree. All the images have been obtained 
by using mechanically cut $\mathrm{Pt} / \mathrm{Rh}(13 \%)$ tips. The map of the local potential in a current-carrying film and the simultaneous information on the topography are obtained in an STP setup that essentially follows the double-feedback technique [6]. A schematic diagram of the setup is shown in Fig. 1. In this technique the sample is biased such that a spatially uniform a.c. voltage (applied between the tip and the sample) is superposed on a spatially varying d.c. (applied across the length of the sample). The a.c. tunnel current is used for topographic imaging, using a lock-in amplifier and a feedback which controls the $Z$-axis as in conventional topography. The lock-in amplifier serves as a synchronous demodulator. The biasing circuit is such that the two ends of the sample are at the same a.c. potential while there is a d.c. bias across its length. The d.c. tunnel current is used to null the local potential at the site of the tip using an integrator, thus giving a zero current detection of the local potential gradients. Both a.c. and d.c. biasing circuits are floating. Since the tip is held at virtual ground by the current amplifier, it ensures a zero d.c. voltage drop across the tunnel junction. The integrator voltage thus provides a direct measurement of the local potential $\phi(x, y)$ due to the applied d.c. bias at the site of the tip. The cancellation of the d.c. bias between the tip and the sample is essential in order to see the small variations in the local potential. The technique is limited by the noise present in the system and on the resolution of the analog-to-digital converter (ADC) card used for data acquisition. In its present form using a 16-bit ADC we can resolve variations in the chemical potential of $100 \mu \mathrm{eV}$. The proper operation of the double-feedback circuit needs somewhat critical adjustments of different time constants. For an a.c. frequency of $500 \mathrm{~Hz}$, the lock-in time constant used was $4 \mathrm{~ms}$. The topographic feedback circuit integrator has a time constant of 2-100 ms and is usually operated at $2 \mathrm{~ms}$. The potential feedback circuit has an integrator time constant of 5.6-27 ms. While operating the two feedbacks simultaneously it is ensured that the time

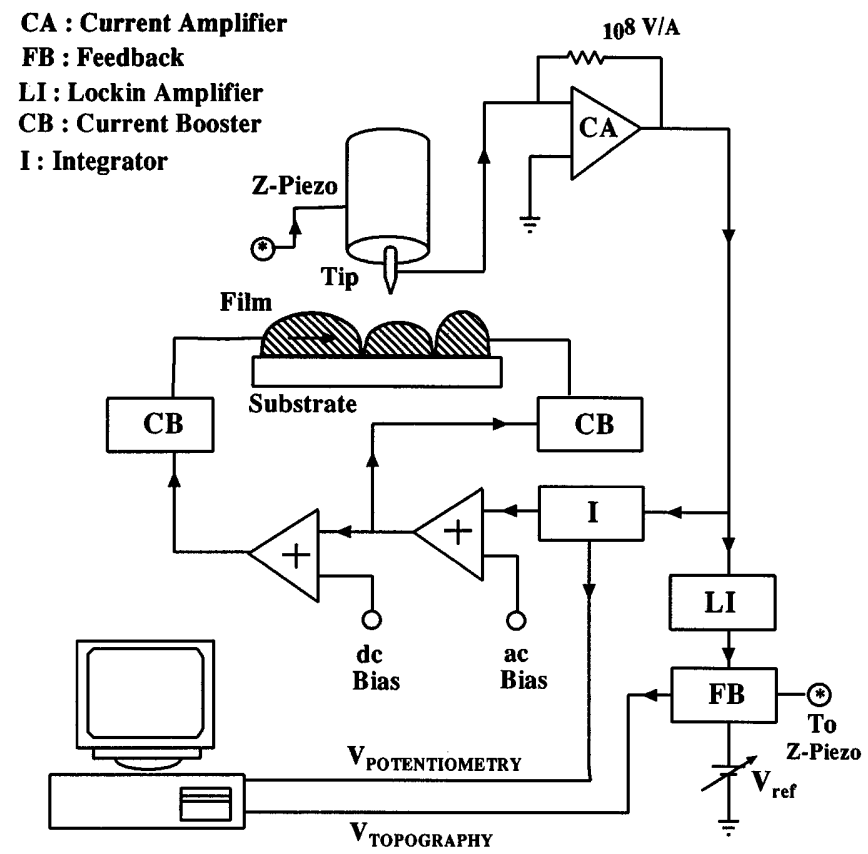

Fig. 1. Schematic of the STM and the STP setup used in the experiment constants are at least separated by a factor of 5-7 in order to prevent cross-talk between them.

We have performed STM/STP measurements on various metal films of, for example, gold, copper, and platinum. Previous studies on metal oxide chemical vapor deposition (MOCVD) grown copper films by us have shown that under certain conditions local nanostructure severely affects the macroscopic resistivity $[12,13]$. In this short report we only present our results on STM and STP investigations on thin platinum films, which were done in order to study the influence of localized scatterers on local potentials and fields. Platinum was chosen as it does not oxidize at any temperature in air [14] and hence makes the interpretation of the observed surface potentials easier. The experiments were performed on thin platinum films $(\approx 10 \mathrm{~nm})$ which were deposited on precleaned glass substrates by electron-beam (e-beam) evaporation in an oil-free evaporation chamber at a base pressure of $5 \times 10^{-8}$ torr. The vacuum chamber was pumped by a turbomolecular pump backed by a diaphragm pump resulting in an oil-free environment. The platinum (99.9999\%) was evaporated from a graphite crucible at a typical rate of $1.5 \mathrm{~nm} / \mathrm{min}$. The substrate was at a distance $\approx 15 \mathrm{~cm}$ vertically above the source. The STM and STP measurements were performed under ambient conditions.

To characterize the films, four-probe resistivity $(\rho)$ measurements were performed on the film from room temperature down to $4.2 \mathrm{~K}$. The films have a rather high room-temperature $\rho$ of $\approx 178 \mu \Omega \mathrm{cm}$ and a residual resistivity of $\approx 145 \mu \Omega \mathrm{cm}$. The residual resistivity ratio is $\approx 1.23$. The reason for the choice of a highly resistive film will be explained later on. Figure 2 shows the variation of the film resistance $R$ with temperature. The average mean free path estimated from the room-temperature resistivity is $\approx 0.22 \mathrm{~nm}$ which is comparable to the lattice spacing in $\mathrm{Pt}$ and much smaller than the average grain size of $10 \mathrm{~nm}$ obtained by STM. This implies that the electrons in the metal film are on the verge of localization within a grain although they can still be regarded

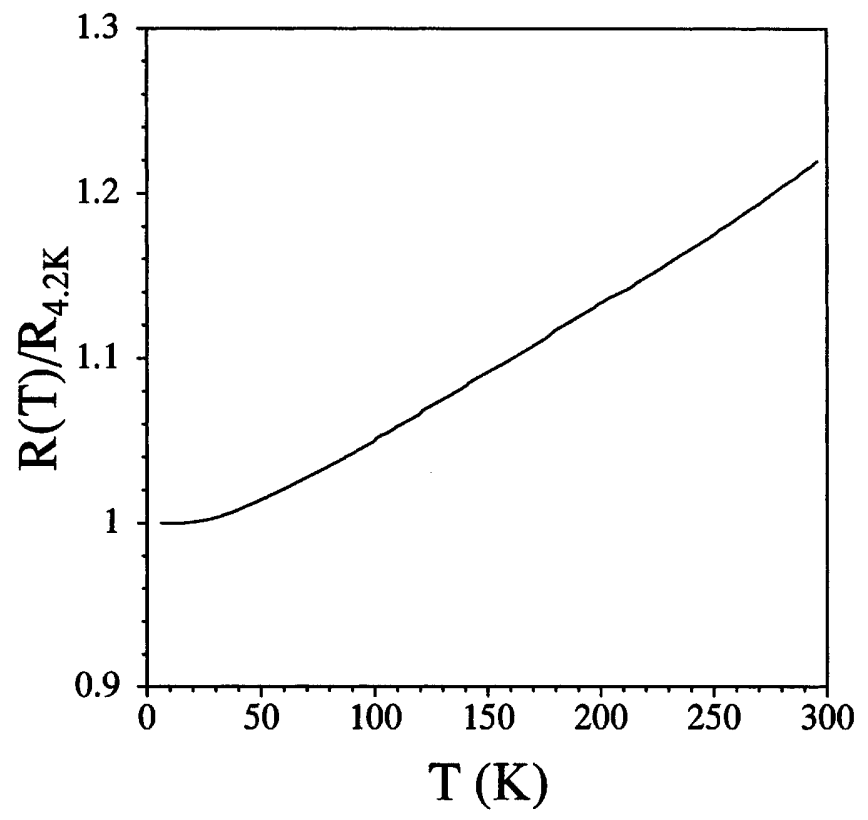

Fig. 2. Temperature dependence of the resistance of the Pt film 
as conducting through an extended state. The reasons for the high $\rho$ are small thickness of the film and the considerable roughness of the glass substrate on which the film is deposited. The substrate had an $\mathrm{rms}$ roughness of $\approx \pm 1.8 \mathrm{~nm}$ as measured over a $700 \mathrm{~nm} \times 700 \mathrm{~nm}$ region by an atomic force microscope (AFM).

\section{Results and discussion}

Figure 3 shows the results of simultaneous topographic and potentiometric measurements in a $40 \mathrm{~nm} \times 40 \mathrm{~nm}$ region of the film. The topographic image in Fig. 3a was obtained with an ac. bias of $20 \mathrm{mV} \mathrm{rms}$ at a frequency of $500 \mathrm{~Hz}$ and with an rms tunnel current of $0.3 \mathrm{nA}$ (effective tunneling resistance of the tip-substrate junction was $\approx 60 \mathrm{M} \Omega$ ). The images were recorded with a scan time of $30 \mathrm{~s}$ and a typical lock-in time constant of $4 \mathrm{~ms}$. Figure $3 \mathrm{~b}$ shows the corresponding potentiometric image. A d.c. bias was applied across a thin length of the film giving a macroscopic field of $13 \mathrm{~V} / \mathrm{cm}$ and a macroscopic current density $\boldsymbol{j} \approx 10^{5} \mathrm{~A} / \mathrm{cm}^{2}$. The direction of the macroscopic $j$ was kept at $110^{\circ}$ to the fast scan direction ( $X$ axis in our images) to avoid any artifacts that might arise if the current direction and the scan direction are the same. This is indicated by an arrow in Fig. 3b The potential image from black to white covers a range of $40 \mathrm{mV}$, with the dark regions representing a lower electrochemical potential than the brighter regions.

From the STP image in Fig. 3b it is immediately apparent that the potential does not drop uniformly across the film surface. Jumps in the potential occur at points where there is a GB. The drop in the potential, interestingly, spreads over a scale of nearly $20 \mathrm{~nm}$. This seems larger than the screening length expected from the Thomas-Fermi approximation. It is, however, not clear at this moment whether it is correct to expect a screening length at the GB to be the same as that in the bulk. It is evident that the field and current density across the film are not uniform and the field is highly concentrated in
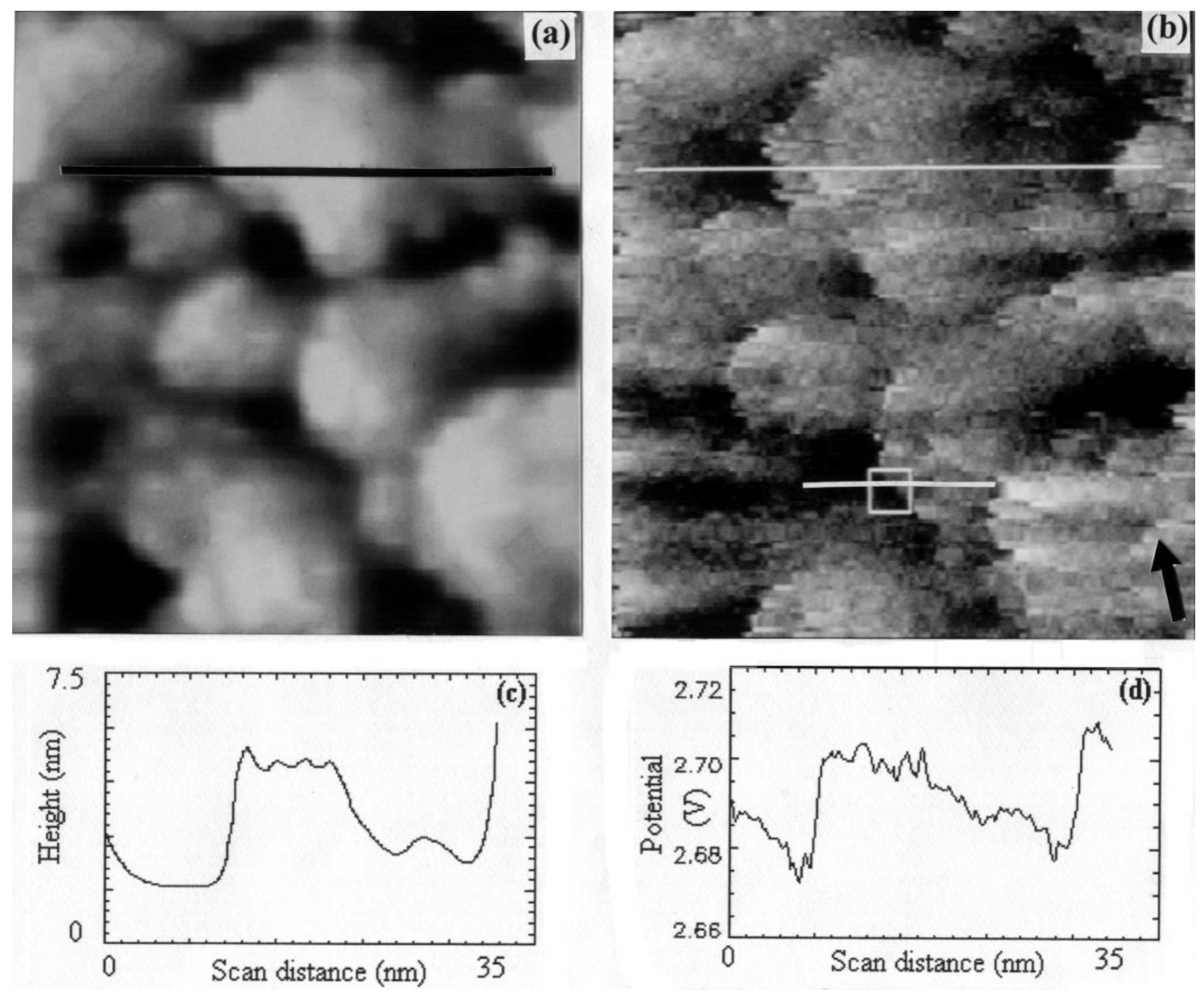

Fig. 3. a STM and b STP images of the same region of the film. The scan size is $40 \mathrm{~nm} \times 40 \mathrm{~nm}$. The profiles taken over the region marked by the lines on the upper part of the images are shown in $\mathbf{c}$ for the STM image and $\mathbf{d}$ for the STP image. The direction of the bulk transport current is marked by an arrow in the lower right corner in $\mathbf{b}$ 
regions where the scattering actually occurs, resulting in significant deviations from the average macroscopic potential. Figures $3 \mathrm{c}, \mathrm{d}$ show line profiles across the same regions as in Fig. 3a,b respectively. It is clearly seen that there is a one to one correspondence between the GBs in the topographic image and the voltage drops in the potential image. It is to be noted that in the absence of a d.c. bias across the sample, no such voltage drops are observed in the potential image and it is flat and featureless. This shows that these jumps in the potential image are the result of an actual build up of a field near a GB. The voltage jumps can be as high as $10 \mathrm{mV}$ occurring over a distance scale of $1 \mathrm{~nm}$.

The local transport field in the surface plane is given by $\boldsymbol{E}_{\|}(x, y)=-\operatorname{grad}[\phi(x, y)]$. Figure 4a shows the $E_{\|}$field obtained from the potential distribution in the region of a single GB marked by a square in Fig. 3b. The gradient has been taken after a smoothing of two neighboring data points. The length of the arrows and the head indicate the strength and direction of the field. It shows that the field is concentrated in
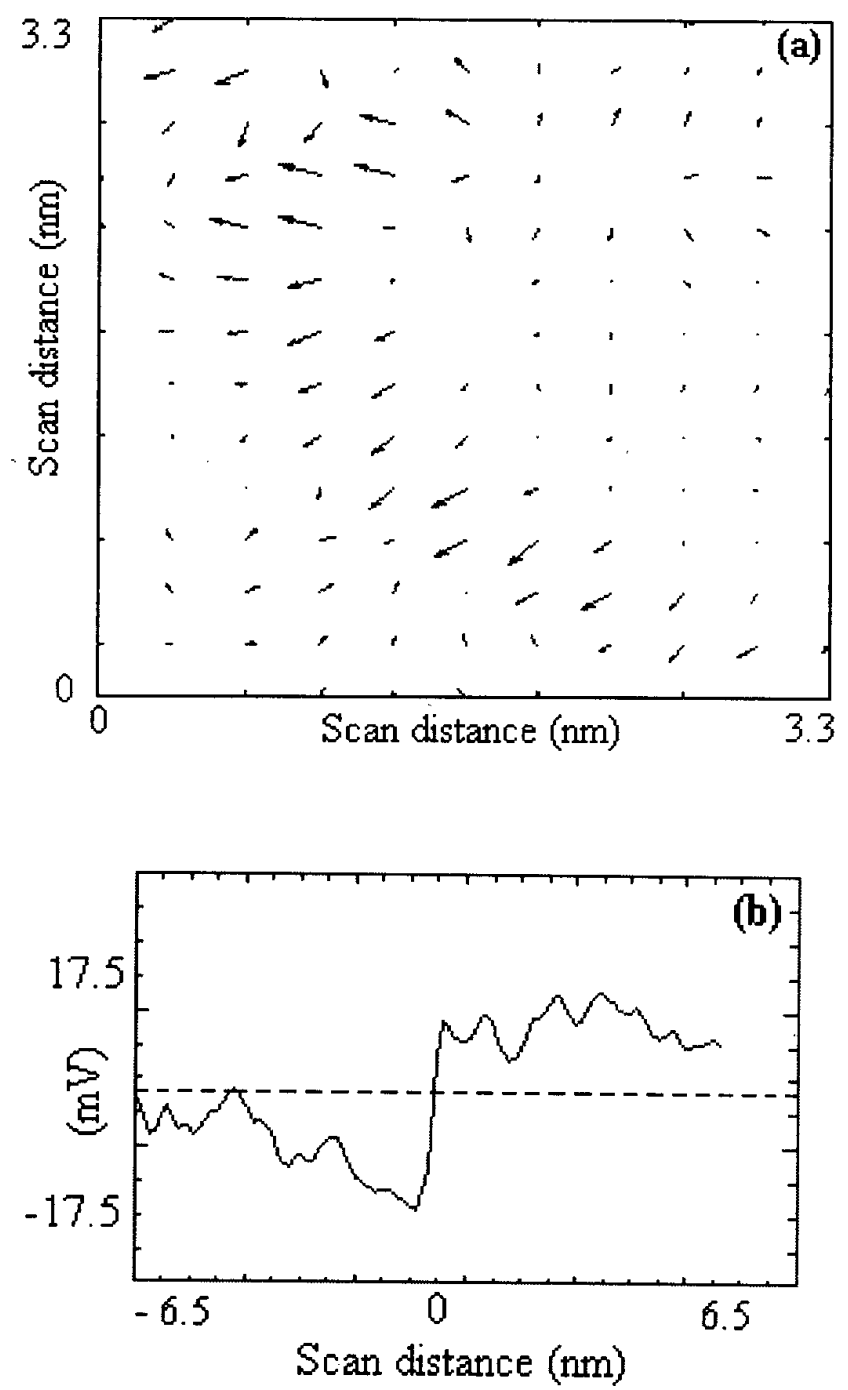

Fig. 4. a A map of the electric field evaluated in a region around a GB. This region $(3.3 \mathrm{~nm} \times 3.3 \mathrm{~nm})$ is marked as a box in Fig. $3 b$ The direction of the arrow is the local field direction and the size is a scaled measure of the magnitude. b A line profile over the boxed region in the STP image Fig. $3 b$ the region of the scatterer and is very low in the interior of the grain. The magnitudes of the local field at the GBs can be as high as $10^{4}-10^{5} \mathrm{~V} / \mathrm{cm}$. Figure $4 \mathrm{~b}$ is a line scan in the same region with the d.c. background removed.

The exact direction of the local current flow at the GBs depends on the GB structure, the connectivity between the grains and the degree of misorientation between the neighboring grains. Also the local resistivity around a GB can be substantially different from that of the bulk. Relatively little work has been done on the electronic properties of GBs in metals as against semiconductors [15-17]. In semiconductors it is known that in the case of a tilt boundary or any other distribution of dislocations with a preferred orientation, the conductivity along the dislocation lines is easier than perpendicular to them. For the metallic case it can be entirely different.

In a semicontinuous film like our platinum film which has a number of voids and a very granular structure it is very difficult to hypothesize how exactly the GB would affect the local electric field. From our potentiometric images we find that the field at the GB is spatially very inhomogeneous. In general it is a maximum perpendicular to the GB, irrespective of the direction of the macroscopic field. This is brought out by the field lines in Fig. 4a. As the conductivity varies across the boundary in the range of a few nanometers it is difficult to predict the exact path of the local current.

The average field in this scan range $(40 \mathrm{~nm} \times 40 \mathrm{~nm})$ calculated from the gradients of the potential is $\approx 25 \mathrm{~V} / \mathrm{cm}$ which compares well with the macroscopic field of $13 \mathrm{~V} / \mathrm{cm}$. The direction of the average field is $\approx 66^{\circ}$ to the fast scan direction. Thus the field direction is severely altered by the effect of the localized scatterers. It is worth noting that the averaging of the local field over a large range (compared to the grain size) is needed to get the average bulk field.

It was argued by some researchers whether artifacts from tips can mask the actual potentiometric features. We have considered these issues. As mentioned before the potential image is flat and featureless when there is no d.c. current through the sample. Also the features are seen to scale appropriately with different scan sizes and are repeatedly observed over several scans and scan times. If the features were due to tip convolution effects or capacitative coupling between the two feedbacks then the features would change with scan time and size. Our choice of a somewhat resistive but metallic film is also important because it does produce large fields at the GBs which are weakly connected. At the same time the film is not insulating and hence the transport from grain to grain is not by tunneling or hopping. In such a case the effect of charging of individual grains would be seen.

The high field build up across the GB is due to poor connectivity between the grains resulting in a large GB reflection coefficient. The reflection coefficient, $R_{\mathrm{g}}$, can be estimated from a knowledge of the mean free path, the voltage drop at the boundary and the electric field component perpendicular to the grain $[11,18]$. We estimated the values of $R_{\mathrm{g}}$ at different points for several grains. The values lie between 0.5 to 0.7 . We were able to estimate an average bulk $\left\langle R_{\mathrm{g}}\right\rangle \approx 0.9$ assuming that the resistivity arises completely from the GB scattering $[12,13]$. This gives an upper limit on $\left\langle R_{\mathrm{g}}\right\rangle$. The higher value of the average macroscopic $\left\langle R_{\mathrm{g}}\right\rangle$ (as compared to the microscopic $R_{\mathrm{g}}$ ) suggests that the GB is not the only source of high resistivity. Also there is a distinction between 
the two $R_{\mathrm{g}}$ values. To estimate the microscopic $R_{\mathrm{g}}$ we use the potential gradient which is a two-dimensional (2D) gradient evaluated on the surface. The grains have a substantial connectivity along the depth and the true three-dimensional (3D) potential gradient can be different. This would modify the $R_{\mathrm{g}}$ obtained from simple 2D potentials.

\section{Summary and conclusion}

In summary we have presented the study of the local potentials and fields on a metallic but resistive platinum film using the STM and STP. It is seen from the above discussion that in an inhomogeneous system like a polycrystalline metal film, the electric field at the nanoscale is very inhomogeneous and the electric field at the GBs is maximum perpendicular to the boundary. In these highly resistive films GB scattering leads to a significant enhancement of the $\rho$ of the films. We were able to clearly visualize the build up of an electric field arising from scattering at the GBs and evaluate the value of the GB reflection coefficient at individual GBs and also an averaged reflection coefficient value from the resistivity measurements.

Acknowledgements. We would like to thank Professor N. Chandrasekhar for his support in depositing the film and DST for partial financial help. AKR wants to thank CSIR for financial support through a sponsored scheme.

\section{References}

1. R. Landauer: IBM J. Res. Dev. 1, 223 (1957)

2. R. Landauer: Phys. Rev. B 14, 1474 (1976)

3. R.S. Sorbello: Phys. Rev. B 23, 5119 (1981)

4. G. Binning, H. Rohrer, C. Gerber, E. Weibel: Phys. Rev. Lett. 49, 57 (1982)

5. G. Binning, C.F. Quate, C. Gerber: Phys. Rev. Lett. 56, 930 (1986)

6. P. Muralt, D.W. Pohl: Appl. Phys. Lett. 48, 514 (1986)

7. J.R. Kirtley, S. Washburn, M.J. Brady: Phys. Rev. Lett. 60, 1546 (1988)

8. J.P. Pelz, R.H. Koch: Rev. Sci. Instrum. 60, 301 (1989)

9. C.S. Chu, R.S. Sorbello: Phys. Rev. B 40, 5950 (1989)

10. B.G. Briner, R.M. Feenstra, T.P. Chin, J.M. Woodall: Phys. Rev. B 54, R5283 (1996)

11. M.A. Schneider, M. Wenderoth, A.J. Heinrich, M.A. Rosentreter, R.G. Ulbrich: Appl. Phys. Lett. 69, 1327 (1996)

12. G. Ramaswamy, A.K. Raychaudhuri, J. Goswami, S.A. Shivashankar: J. Phys. D: Appl. Phys. 30, L5 (1997)

13. G. Ramaswamy, A.K. Raychaudhuri, J. Goswami, S.A. Shivashankar: J. Appl. Phys. 82, 3797 (1997)

14. CRC Handbook of Physics and Chemistry, ed. D.R. Lide (CRC Press, Boca Raton, FL 1994) pp. 4-21

15. S. Amelinckx, W. Dekeyser: In The Structure and Properties of Grain Boundaries, Solid State Physics, ed. by F. Seitz, D. Turnbull, Vol. 8 (Academic Press, New York 1959) p. 327

16. H.F. Matare: J. Appl. Phys. 56, 2605 (1984)

17. J. Thibault, J.L. Rouviere, A. Bourret: In Grain Boundary in Semiconductors, Materials Science and Technology, ed. by R.W. Cahn, P. Haasen, E.J. Kramer, Vol. 4 (VCH, Weinheim 1991) p. 321

18. A. Knabchen: J. Phys.: Condens. Matter 3, 6989 (1991) 